\title{
Suppression of superconductivity by two-channel Kondo impurities
}

\author{
K.-H. Luk \\ Centre of Computing Services and Telecommunications, The Hong Kong University of Science and Technology, \\ Clear Water Bay, Kowloon, Hong Kong \\ and Department of Physics, The Ohio State University, Columbus, Ohio 43210 \\ M. Jarrell \\ Department of Physics, ML-0011, University of Cincinnati, Cincinnati, Ohio 25221-0011 \\ D. L. Cox \\ Department of Physics, The Ohio State University, Columbus, Ohio 43210 \\ (Received 28 April 1994; revised manuscript received 5 July 1994)
}

\begin{abstract}
We have performed a calculation on the change of the critical temperature $T_{c}$ of a superconducting host with respect to the change of the concentration $c$ of two-channel Kondo impurities in the limit of vanishing $c . T_{c}$ suppressions are found for both spin-singlet flavor-triplet and spin-triplet flavor-singlet pairings for temperatures as low as $T_{K} / T_{c 0}>1$, where $T_{K}$ and $T_{c 0}$ are the Kondo temperature and the critical temperature of the host in the absence of impurities, respectively. The suppression is much larger for the spin-singlet flavor-triplet pairing. We also found empirically that $\left(\partial T_{c} / \partial c\right)_{c=0}$ can be expressed as a product of two functions $g\left(T_{K} / T\right)$ and $f\left(\lambda_{0}, \omega_{0}\right)$, where $\lambda_{0}$ and $\omega_{0}$ are electron phonon coupling and Einstein frequency, respectively.
\end{abstract}

\section{INTRODUCTION}

Considerable attention has been given recently to the physics of the two-channel Kondo model

$$
H_{\mathrm{tck}}=\sum_{\mathrm{p}, m, \alpha} \epsilon_{\mathrm{p}} c_{\mathrm{p} m \alpha}^{\dagger} c_{\mathrm{p} m \alpha}+J \sum_{\mathrm{p}, \mathrm{p}^{\prime}, m, \alpha, \beta} \mathbf{S} \cdot \sigma_{\alpha \beta} c_{\mathrm{p} m \alpha}^{\dagger} c_{\mathrm{p}^{\prime} m \beta} \text {. }
$$

In this model, the operator $c_{\mathrm{p} m \alpha}^{\dagger}$ creates a conduction electron of momentum $\mathbf{p}$, orbital channel $m$, and spin projection $\alpha$, and $\sigma$ is the spin $-\frac{1}{2}$ Pauli matrix. Here the channel index $m$ runs from 1 to 2 . The electrons, described by the flavor $m$ and spin $\alpha$, interact with a flavorless impurity spin $\mathbf{S}=\frac{1}{2}$. The two-channel Kondo Hamiltonian could arise from magnetic impurities embedded in a host metal when the orbital structure of the impurity is taken into account. ${ }^{1}$ The channel quantum number is then the label for orbital quantum number or the label for partners of the symmetry representation of the groundstate electron. $^{2}$

The current interest in the model stems both from recent theoretical advances using conformal field theory, ${ }^{3}$ bosonization, ${ }^{4}$ and large- $N$ methods ${ }^{5,6}$ as well as from experimental observation of possible two-channel Kondo physics in the alloys $\mathrm{Y}_{1-x} \mathrm{U}_{x} \mathrm{Pd}_{3},{ }^{7} \mathrm{Th}_{1-x} \mathrm{U}_{x} \mathrm{Ru}_{2} \mathrm{Si}_{2},{ }^{8}$ $\mathrm{La}_{1-x} \mathrm{Ce}_{x} \mathrm{Cu}_{2.2} \mathrm{Si}_{2},{ }^{9}$ and in nanoscale point contact devices. ${ }^{10,11}$ In the case of the $U$ ions, an explanation has been put forward in terms of the quadrupolar Kondo effect where quantum shape fluctuations between two equivalent states of the $5 f^{2}$ configuration on the actinide ions are screened by orbital motion of conduction electrons in degenerate magnetic states, so that the channel index is the local magnetic index. ${ }^{12}$ For the Ce ions, a magnetic two-channel Kondo effect in which the channel label becomes a local orbital index has been proposed. ${ }^{13}$ For the point contacts, a two-level system Kondo effect ${ }^{14}$ has been proposed in which an atom tunnels between two equivalent sites (the site index becomes the impurity spin index) with electronic assistance; as for the quadrupole Kondo effect, the channel index is the magnetic label of the conduction electrons and conduction orbital motion screens the atomic hops between sites.

The physics of the two-channel Kondo Hamiltonian is very different from that of the single-channel Kondo Hamiltonian. The most important point of the twochannel Hamiltonian is that the impurity spin is overscreened by the conduction electrons at low temperatures, and thus it induces an antiferromagnetic interaction between the remaining free conduction electrons and the overscreened cloud around the impurity spin. This overscreening leads to a nontrivial stable fixed point at a finite value of $J$. As a result, all the non-Fermi-liquid behavior is exhibited in physical quantities. The ground state is degenerate with nonzero fraction entropy of the impurity, $S_{\text {imp }}=R \ln 2 / 2$, where $R$ is the universal gas constant. The magnetic susceptibility and specific-heat coefficient show logarithmic divergence as the temperature goes to zero. ${ }^{15,16}$

Using conformal field theory methods, Affleck and Ludwig have studied the flavor-singlet, spin-singlet, oddin-radial-parity pair field correlations about the twochannel impurity, ${ }^{3(a)}$ while Emery and Kivelson have used Abelian bosonization methods to study the corresponding odd-in-time-reversal pair field correlations, ${ }^{4}$ subsequently studied with conformal theory. ${ }^{3(b)}$ Each study takes into account spin and flavor degrees of freedom, and the resulting local pair field susceptibilities are 
predicted to diverge logarithmically in the temperature $T$ as $T \rightarrow 0$. However, the flavor-singlet, spin-triplet and flavor-triplet, spin-singlet pair field susceptibilities do not diverge for $T \rightarrow 0 .{ }^{3,4}$ The rate of change of transition temperature with respect to the change of two-channel Kondo impurity concentration, $\left(\partial T_{c} / \partial c\right)_{c=0}$, was estimated approximately by $\operatorname{Cox}^{17}$ and Tsvelik, ${ }^{18}$ and an enhancement in $T_{c}$ was found. These authors modeled the interaction by the dynamic two-channel impurity susceptibility and the two-particle impurity Green's function, and the pair-breaking effect was neglected in their models. The main purpose of the present work is to find $\left(\partial T_{c} / \partial c\right)_{c=0}$ of the host superconductor for two-channel Kondo impurities using the quantum Monte Carlo technique combined with the Eliashberg formalism.

An outline of our paper is as follows. We introduce the Hamiltonian of the superconducting host with twochannel Kondo impurities randomly distributed in the system in Sec. II. Quantum Monte Carlo measurement of the self-energy and effective interaction of the phonon and impurity are then discussed in Secs. III and IV, respectively. In Sec. $\mathrm{V}$, we derive formulas for calculating $T_{c}$ and for the change in $T_{c}$ with respect to a change in concentration $c$ in the limit the concentration approaches zero. Then we provide tests performed on our numerical codes in Sec. VI. The results are then presented in Sec. VII, which is followed by a conclusion. We note that our approach in calculating $T_{c}$ and the change of $T_{c}$ in Eliashberg formalism is identical to those of recent works by Jarrell and co-workers ${ }^{19,20,22,23}$ and Owen and Scalapino. ${ }^{21}$

\section{HOST AND IMPURITY SYSTEM}

The system we are interested in is a superconducting host with two-channel Kondo impurities. We assume the impurities are randomly embedded in the host, and the impurity concentration is so low that the impurities are independent of each other. The first assumption allows us to average any macroscopic quantity over all the configurations of impurities, and it restores the translational symmetry of the system. The second assumption allows an expansion of a macroscopic quantity to the first order in concentration. ${ }^{24}$

The Hamiltonian of the system consists of four parts,

$$
H=H_{\text {el }}+H_{l}+H_{\text {ep }}+H_{\text {imp }} \text {. }
$$

The electron Hamiltonian $H_{\mathrm{el}}$ describes noninteracting electrons and it is given by

$$
H_{\mathrm{el}}=\sum_{\mathrm{p} m \sigma} c_{p} c_{\mathrm{p} m \sigma}^{\dagger} c_{\mathrm{p} m \sigma}
$$

where we assume that $\epsilon_{\mathrm{p}}=\mathrm{p}^{2} / 2 m_{e}$ is the bare freeelectron energy ( $m_{e}$ is the electron mass), $c_{\mathrm{p} m \sigma}\left(c_{\mathrm{p} m \sigma}^{\dagger}\right)$ is the destruction (creation) operator of an electron with momentum p, channel $m$, and spin $\sigma$ with energy $\epsilon_{\mathrm{p}}$. The lattice Hamiltonian $H_{l}$ describes noninteracting phonons given by

$$
H_{l}=\sum_{\mathbf{q} \lambda} h \omega_{\mathbf{q} \lambda}\left(a_{\mathbf{q} \lambda}^{\dagger} a_{\mathbf{q} \lambda}+\frac{1}{2}\right),
$$

where $a_{\mathrm{q} \lambda}\left(a_{\mathrm{q} \lambda}^{\dagger}\right)$ is the destruction (creation) operator of a phonon with vector $q$, polarization $\epsilon_{\mathrm{q} \lambda}$, and bare phonon energy $h \omega_{\mathrm{q} \lambda}$. The electron-phonon Hamiltonian $H_{\mathrm{ep}}$ describes the interaction of electrons with phonons given by

$H_{\mathrm{ep}}=\sum_{\mathrm{pp}^{\prime} m \lambda \sigma} g_{\mathrm{pp}^{\prime} \lambda}\left(a_{\left(\mathrm{p}^{\prime}-\mathrm{p}\right) \lambda}+a_{-\left(\mathrm{p}^{\prime}-\mathrm{p}\right) \lambda}^{\dagger}\right) c_{\mathrm{p}^{\prime} m \sigma^{\prime}}^{\dagger} c_{\mathrm{p} m \sigma}$,

where $g_{\mathrm{pp}^{\prime} \lambda}$ is the electron-phonon coupling strength. For simplicity, we assume the Einstein mode for the phonons with frequency $\omega_{0}$ in the host, and the electronphonon interaction $g_{0}$ to be momentum independent. Generalization of the formalism to a more realistic phonon spectrum is straightforward. However it is believed that the single phonon frequency model captures all the essential physics regarding the quantity $\left(\partial T_{c} / \partial c\right)_{c}=0$. The impurity Hamiltonian $\boldsymbol{H}_{\text {imp }}$ describes the interaction of two-channel Kondo impurities with conduction electrons given by

$$
H_{\mathrm{imp}}=J \sum_{\mathrm{p}, \mathrm{p}^{\prime}, m, \sigma, \sigma^{\prime}, \mathbf{R}_{i}} \mathbf{S}\left(\mathbf{R}_{i}\right) \cdot \sigma_{\sigma \sigma^{\prime}} c_{\mathrm{p} m \sigma^{\prime}}^{\dagger} c_{\mathrm{p}^{\prime} m \sigma^{\prime}} e^{i\left(\mathrm{p}-\mathrm{p}^{\prime}\right) \cdot \mathbf{R}_{i}}
$$

where $\mathbf{S}\left(\mathbf{R}_{i}\right)$ is the impurity spin at random site $\mathbf{R}_{i}$. The effects of the Coulomb interaction are to renormalize the bare free-electron energy and the bare electron-phonon coupling. ${ }^{25,26}$ In addition $g_{0}$ is an adjustable parameter in our $\left(\partial T_{c} / \partial c\right)_{c=0}$ calculation; it is not unrealistic to ignore the Coulomb interaction in our model.

The phonon and electron Green's functions, in the absence of impurity and the interaction between phonon and electron, are given, respectively, by

$$
\begin{aligned}
& D_{0}\left(i v_{m}\right)=\frac{-2 \omega_{0}}{v_{m}^{2}+\omega_{0}^{2}}, \\
& G_{0}\left(\mathrm{p}, i \omega_{n}\right)=\frac{1}{i \omega_{n}-\epsilon_{\mathrm{p}}},
\end{aligned}
$$

where $v_{n}=2 n \pi T, \omega_{n}=(2 n+1) \pi T$, and $T$ is the temperature.

We need both the conduction-electron self-energy and the effective electron-electron interaction for the $T_{c}$ and $\left(\partial T_{c} / \partial c\right)_{c=0}$ calculations. The self-energy and interaction have two contributions, namely phonon and impurity. The contributions of phonons can be found analytically, while the contributions of impurities can be found exactly by quantum Monte Carlo simulation.

\section{SELF-ENERGY}

The dressed electron Green's function is given by

$$
G\left(\mathrm{p}, i \omega_{n}\right)=\frac{1}{i \omega_{n} Z_{n}-\epsilon_{\mathrm{p}}},
$$

where the renormalization factor is defined by

$$
Z_{n}=1-\frac{\Sigma\left(i \omega_{n}\right)}{i \omega_{n}} \text {. }
$$

The self-energy of the electron $\Sigma\left(i \omega_{n}\right)$ has contributions from both phonons and impurities

$$
\Sigma\left(i \omega_{n}\right)=\Sigma_{\mathrm{ph}}\left(i \omega_{n}\right)+\Sigma_{\mathrm{imp}}\left(i \omega_{n}\right) .
$$


The self-energy is essentially momentum independent because the phonon contribution $\Sigma_{\mathrm{ph}}$ is known to be only weakly dependent on the momentum over the range $\epsilon_{\mathrm{p}} \sim \omega_{0},{ }^{28}$ and the impurity contribution $\Sigma_{\mathrm{imp}}$ is momentum independent since the impurity interaction is local.

\section{A. Contribution of phonons}

By Migdal's theorem, the contribution of phonons to the electron self-energy $\Sigma_{\mathrm{ph}}\left(\mathbf{p}, i \omega_{n}\right)$ can be approximated by the single diagram, shown in Fig. 1, to the order $O(m / M)$. Here $m$ and $M$ are the mass of the electron and the nucleus, respectively. ${ }^{27}$ We implicitly assume the difference in quasiparticle energies is much less than the product of the momentum transfer and the Fermi velocity $v_{F}$, i.e.,

$$
\left|\epsilon_{\mathrm{p}}-\epsilon_{\mathbf{p}^{\prime}}\right|<<\left|\mathbf{p}-\mathbf{p}^{\prime}\right| v_{F} .
$$

The phonon Green's function can be expressed in terms of the spectral function $B_{\alpha \beta}(q, \omega)$ as

$$
\begin{aligned}
D_{\alpha \beta}\left(\mathbf{q}, i \omega_{v}\right)=\int_{0}^{\infty} d \omega B_{\alpha \beta}(\mathbf{q}, \omega)[ & \left(i \omega_{v}-\omega\right)^{-1} \\
& \left.-\left(i \omega_{v}+\omega\right)^{-1}\right] .
\end{aligned}
$$

In terms of Green's functions, the $\Sigma_{\mathrm{ep}}\left(\mathbf{p}, i \omega_{n}\right)$ is given by

$$
\begin{aligned}
\Sigma_{\mathrm{ph}}\left(\mathrm{p}, i \omega_{n}\right)=-\frac{1}{\beta} \sum_{\mathrm{p}^{\prime}, n^{\prime}, \alpha, \beta} & g_{\mathrm{pp}^{\prime} \alpha} g_{\mathrm{p}^{\prime} \mathrm{p} \beta} \\
& \times D_{\alpha \beta}\left(\mathbf{p}-\mathbf{p}^{\prime}, i \omega_{n}-i \omega_{n^{\prime}}\right) \\
& \times G\left(\mathbf{p}^{\prime}, i \omega_{n^{\prime}}\right) .
\end{aligned}
$$

Substituting the electron and phonon Green's functions, we have

$$
\begin{aligned}
\Sigma_{\mathrm{ph}}\left(\mathbf{p}, i \omega_{n}\right)=\frac{1}{\beta} \sum_{\alpha \beta \mathbf{p}^{\prime} n^{\prime}} \int_{0}^{\infty} & d \omega g_{\mathrm{pp}^{\prime} \alpha} g_{\mathrm{p}^{\prime} \mathbf{p} \beta} B_{\alpha \beta}\left(\mathbf{p}-\mathbf{p}^{\prime}, \omega\right) \\
& \times \frac{2 \omega}{\left(\omega_{n}-\omega_{n^{\prime}}\right)^{2}+\omega^{2}} \\
& \times \frac{1}{\left(i \omega_{n^{\prime}} Z_{n^{\prime}}-\epsilon_{\mathbf{p}^{\prime}}\right)}
\end{aligned}
$$

The electron-electron spectral function $\alpha^{2} F$ is defined as

$$
\alpha^{2} F\left(\mathbf{p}, \mathbf{p}^{\prime}, \omega_{v}\right)=N(0) \sum_{\alpha \beta} g_{\mathrm{pp}^{\prime} \alpha} g_{\mathrm{p}^{\prime} \mathbf{p}^{\beta}} B_{\alpha \beta}\left(\mathbf{p}-\mathbf{p}^{\prime}, \omega_{v}\right),
$$

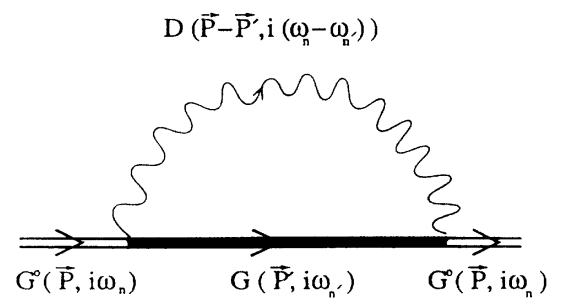

FIG. 1. The phonon contribution of electron self-energy $\Sigma_{\text {ep }}\left(\mathbf{p}, i \omega_{n}\right)$. The phonon and electron Green's functions are interacting Green's functions in the presence of electron-phonon coupling.

$$
\begin{aligned}
\alpha^{2} F\left(\omega_{v}\right) & =\left\langle\alpha^{2} F\left(\mathbf{p}, \mathbf{p}^{\prime}, \omega\right)\right\rangle_{\mathrm{FS}} \\
& =\frac{1}{N(0)^{2}} \sum_{\mathbf{p p}^{\prime}} \alpha^{2} F\left(\mathbf{p}, \mathbf{p}^{\prime}, \omega_{v}\right) \delta\left(\epsilon_{\mathbf{p}}\right) \delta\left(\epsilon_{\mathbf{p}^{\prime}}\right),
\end{aligned}
$$

where $N(0)$ is the density of states at the Fermi surface. Here $\langle\cdots\rangle_{\text {FS }}$ represents an average over the Fermi surface, and energies are measured with respect to the Fermi energy. The function $\alpha^{2} F\left(\mathbf{p}, \mathbf{p}^{\prime}, \omega_{v}\right)$ measures the probability that phonons with frequency $\omega_{v}$ scatter electrons with momentum $\mathbf{p}$ to momentum $\mathbf{p}^{\prime}$, while $\alpha^{2} F\left(\omega_{v}\right)$ measures the probability that phonons with frequency $\omega_{v}$ scatter electrons from one state on the Fermi surface to another. Now we make the Einstein phonon approximation that

$$
\alpha^{2} F(\omega)=N(0) g^{2} \delta\left(\omega-\omega_{0}\right),
$$

and we have

$$
\begin{array}{r}
\Sigma_{\mathrm{ph}}\left(\mathbf{p}, i \omega_{n}\right)=T g^{2} \sum_{n^{\prime}} \int \frac{d^{3} p}{(2 \pi)^{3}} \frac{1}{\left(i \omega_{n^{\prime}} Z_{n^{\prime}}-\epsilon_{\mathrm{p}}\right)} \\
\times \frac{2 \omega_{0}}{\left(\omega_{n}-\omega_{n^{\prime}}\right)^{2}+\omega_{0}^{2}} .
\end{array}
$$

Integrating with respect to the energy and making use of the positivity of the renormalization factor, we have the analytic expression of the self-energy

$$
\Sigma_{\mathrm{ph}}\left(\mathbf{p}, i \omega_{n}\right)=i \operatorname{Tg}^{2} \sum_{n^{\prime}} \frac{2 N(0) \omega_{0} \pi}{\left(\omega_{n}-\omega_{n^{\prime}}\right)^{2}+\omega_{0}^{2}} \operatorname{sgn}\left(\omega_{n^{\prime}}\right) \text {. }
$$

\section{B. Contribution of impurities}

The contribution of impurities to the electron selfenergy $\Sigma_{\text {imp }}$ can be found exactly by the Monte Carlo method. To the first order of impurity concentration $c,{ }^{29}$

$$
\Sigma_{\text {imp }}\left(i \omega_{n}\right)=c T_{\text {imp }}\left(i \omega_{n}\right),
$$

where $T_{\mathrm{imp}}$ is the $t$ matrix, which can be obtained by considering a single impurity through the relation

$G\left(i \omega_{n}\right)=G_{0}\left(i \omega_{n}\right)+G_{0}\left(i \omega_{n}\right) T_{\text {imp }}\left(i \omega_{n}\right) G_{0}\left(i \omega_{n}\right)$.

The impurity site electron Green's function, $G\left(i \omega_{n}\right)$, in the presence of a single two-channel Kondo impurity in the system, can be measured exactly by the quantum Monte Carlo method, ${ }^{30}$ and thus $T_{\text {imp }}$ can be found by inverting the equation.

\section{EFFECTIVE ELECTRON-ELECTRON INTERACTION}

The effective electron-electron interaction also consists of phonon and impurity contributions given by

$$
V_{\text {eff }}\left(\omega_{n}, \omega_{m}\right)=V_{\mathrm{ph}}\left(\omega_{n}, \omega_{m}\right)+V_{\mathrm{imp}}\left(\omega_{n}, \omega_{m}\right) .
$$

The phonon contribution can be calculated analytically, and the impurity contribution can be obtained by the Monte Carlo method. 


\section{A. Contribution of phonon}

The phonon contribution of the effective electronelectron interaction can be found in terms of Matsubara frequency by considering the diagram in Fig. 2. By Migdal's theorem, the contribution of the diagram is exact to the order of $O(m / M)$, where $m$ and $M$ are the electron mass and nucleus mass, respectively. Thus $V_{\mathrm{ph}}$ is given by

$$
V_{\mathrm{ph}}\left(\omega_{n}, \omega_{m}\right)=\frac{-2 \omega_{0} g_{0}^{2}}{\left(\omega_{n}-\omega_{m}\right)^{2}+\omega_{0}^{2}} .
$$

In terms of the quasiparticle energy, the phonon contribution to the electron-electron interaction can be found by substituting

$$
\begin{aligned}
& \epsilon_{\mathbf{k}}=i \omega_{n}, \\
& \epsilon_{\mathbf{k}-\mathrm{q}}=i \omega_{m},
\end{aligned}
$$

and the interaction is given by

$$
V_{\mathrm{ph}}(\mathbf{k}, \mathbf{k}-\mathbf{q})=\frac{2 \omega_{0} g_{0}^{2}}{\left(\epsilon_{\mathbf{k}}-\epsilon_{\mathbf{k}-\mathrm{q}}\right)^{2}-\omega_{0}^{2}} .
$$

Note the interaction $V_{\mathrm{ph}}(\mathbf{k}, \mathbf{k}-\mathbf{q})$ is identical to the one found by the canonical transformation in which the firstorder term in electron-phonon coupling is eliminated. ${ }^{31,32}$ Thus $V_{\mathrm{ph}}$ is attractive for $\left|\epsilon_{\mathrm{k}}-\epsilon_{\mathrm{k}-\mathrm{q}}\right|<\omega_{0}$, and repulsive otherwise. The interaction is attractive because the first electron pulls the positive ions closer to each other so that the potential is lower around the distortion. The second electron takes advantage of the potential and is drawn near the ions. The effect is that two electrons lower their energies by staying closer to the ions, but the interaction is retarded.

\section{B. Contribution of impurity}

In addition to the phonon contribution, the impurity induces an effective electron-electron interaction by polarization. In the single-particle Green's function, the self-energy specifies the effective one-body potential for a particle propagating in a many-body system. Similarly, in the two-particle Green's function the irreducible vertex specifies the effective two-body interaction between two particles propagating in a many-body system. ${ }^{33}$ In the context of Fermi-liquid theory, Landau was able to establish the direct relationship between the irreducible vertex of two-particle Green's function and the quasiparticle interaction parameters. ${ }^{34}$

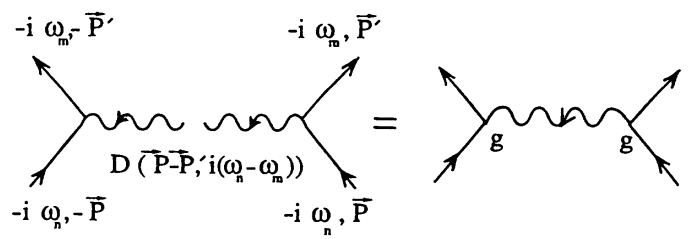

FIG. 2. The electron-electron interaction induced by the phonon.
The local two-particle Green's function in the frequency space is defined by

$$
\begin{aligned}
G_{\alpha}^{(2)}\left(\omega_{n}, \omega_{m}\right)=\int_{0}^{\beta} & d \tau_{1} \cdots d \tau_{4} e^{i \omega_{m}\left(\tau_{1}-\tau_{2}\right)-i \omega_{n}\left(\tau_{3}-\tau_{4}\right)} \\
& \times\left\langle T_{\tau} \Delta_{\alpha}\left(\tau_{1}, \tau_{2}\right) \Delta_{\alpha}^{\dagger}\left(\tau_{3}, \tau_{4}\right)\right\rangle
\end{aligned}
$$

where the label $\alpha$ denotes the pairing operator. We are interested in the flavor-singlet spin-triplet operators defined by

$\Delta_{s+}\left(\tau, \tau^{\prime}\right)=c_{1 \uparrow}(\tau) c_{2 \uparrow}\left(\tau^{\prime}\right)$

$\Delta_{s 0}\left(\tau, \tau^{\prime}\right)=\frac{1}{\sqrt{2}}\left[c_{1 \uparrow}(\tau) c_{2 \downarrow}\left(\tau^{\prime}\right)+c_{1 \downarrow}(\tau) c_{2 \uparrow}\left(\tau^{\prime}\right)\right]$

$\Delta_{s-}\left(\tau, \tau^{\prime}\right)=c_{1 \downarrow}(\tau) c_{2 \downarrow}\left(\tau^{\prime}\right)$,

and the flavor-triplet spin-singlet operators defined by

$$
\begin{aligned}
& \Delta_{f+}\left(\tau, \tau^{\prime}\right)=c_{1 \uparrow}(\tau) c_{1 \downarrow}\left(\tau^{\prime}\right), \\
& \Delta_{f 0}\left(\tau, \tau^{\prime}\right)=\frac{1}{\sqrt{2}}\left[c_{1 \uparrow}(\tau) c_{2 \downarrow}\left(\tau^{\prime}\right)+c_{2 \uparrow}(\tau) c_{1 \downarrow}\left(\tau^{\prime}\right)\right], \\
& \Delta_{f-}\left(\tau, \tau^{\prime}\right)=c_{2 \uparrow}(\tau) c_{2 \downarrow}\left(\tau^{\prime}\right) .
\end{aligned}
$$

Here $c_{i \gamma}$ is the electron destruction operator for flavor $i$ and spin $\gamma$ at the origin defined by

$$
c_{i \gamma}=\int \frac{d^{3} p}{(2 \pi)^{3}} c_{\mathrm{p} i \gamma} .
$$

For integer $m$, the Matsubara frequency $\omega_{m}=(2 m+1) / T$. The interaction $\Gamma_{\alpha}\left(i \omega_{n}, i \omega_{m}\right)$ is related to the corresponding two-particle Green's function $G_{\alpha}^{(2)}\left(i \omega_{n}, i \omega_{m}\right)$ by $^{35}$

$$
\begin{aligned}
G_{\alpha}^{(2)}\left(i \omega_{n}, i \omega_{m}\right)= & \beta^{2} G\left(i \omega_{n}\right) G\left(-i \omega_{n}\right) \delta_{\omega_{n}, \omega_{m}} \\
& -\beta\left|G\left(i \omega_{n}\right) G\left(i \omega_{m}\right)\right|^{2} \Gamma_{\alpha}\left(i \omega_{n} ; i \omega_{m}\right) .
\end{aligned}
$$

Since quantum Monte Carlo simulation measures the two-particle Green's function $G_{\alpha}^{(2)}\left(i \omega_{n}, i \omega_{m}\right)$ and the single-particle Green's function $G\left(i \omega_{n}\right)$, we can extract the effective interaction by inverting the above equation. To the first order in concentration $c$, the integration is given $b^{24}$

$$
V_{\mathrm{imp}}\left(\omega_{n}, \omega_{m}\right)=c \Gamma_{\alpha}\left(i \omega_{n} i \omega_{m}\right) .
$$

\section{V. $T_{c}$ SUPPRESSION}

We search for the signature of superconductivity of the superconducting host in the presence of two-channel Kondo impurities by investigating the divergence of the static long-wavelength pairing susceptibility when the temperature is lowered. In particular, we are interested in the zero-frequency flavor-triplet spin-singlet and flavor-singlet spin-triplet pairing susceptibilities defined, respectively, by 


$$
\begin{aligned}
& \chi_{\mathrm{pair}}^{f+}\left(\omega_{n}=0\right)=\int_{0}^{\beta} \sum_{\mathbf{p}, \mathbf{p}^{\prime}}\left\langle c_{\mathbf{p} 1 \uparrow}(\tau) c_{-\mathbf{p} 1 \downarrow}(\tau)\right. \\
&\left.\times c_{-\mathbf{p}^{\prime} 1 \downarrow}^{\dagger}(0) c_{\mathbf{p}^{\prime} 1 \uparrow}^{\dagger}(0)\right\rangle d \tau, \\
& \chi_{\text {pair }}^{s+}\left(\omega_{n}=0\right)=\int_{0}^{\beta} \sum_{\mathbf{p}, \mathbf{p}^{\prime}}\left\langle c_{\mathrm{p} 2 \uparrow}(\tau) c_{-\mathbf{p} 1 \uparrow}(\tau)\right. \\
&\left.\quad \times c_{-\mathbf{p}^{\prime} 1 \uparrow}^{\dagger}(0) c_{\mathrm{p}^{\prime} 2 \uparrow}^{\dagger}(0)\right\rangle d \tau .
\end{aligned}
$$

In the imaginary time space, the susceptibility is a measure of the probability amplitude of finding a pair of electrons at time $\tau$ given that the pair was created at time 0 . When the system undergoes a superconducting transition, Cooper pairs will be formed spontaneously. An infinitesimal perturbation (injection of Cooper pairs) would lead to the divergence of the static response function $\chi_{\text {pair }}\left(\omega_{n}=0\right)$. This is analogous to the magnetic instability in which the magnetic susceptibility diverges at a critical temperature. In the following, we derive the $T_{c}$ equation and $\left(\partial T_{c} / \partial c\right)_{c=0}$ formula within the Eliashberg formalism, which takes into account the retardation effect of the interaction.

\section{A. $T_{c}$ equation}

To find the $T_{c}$ equation, it is sufficient to consider a particular series of ladder diagrams of $\chi_{\text {pair }}$ (Fig. 3). Given the initial pair, the ladder diagrams allow sampling of other pair configurations under the influence of attractive interaction. Note that in the diagrams, both the electron Green's function and the interaction are dressed with impurity. If we define the function $\phi$ and the matrix $A$ with elements by

$$
\begin{aligned}
& \phi(n)=\int \frac{d^{3} p}{(2 \pi)^{3}} G\left(\mathbf{p}, i \omega_{n}\right) G\left(-\mathbf{p},-i \omega_{n}\right), \\
& \begin{aligned}
A(m, n)=-T \int \frac{d^{3} p}{(2 \pi)^{3}} V_{\mathrm{eff}}\left(\omega_{m}, \omega_{n}\right) \\
\quad \times G\left(\mathbf{p}, i \omega_{n}\right) G\left(-\mathbf{p},-i \omega_{n}\right),
\end{aligned}
\end{aligned}
$$

then the geometric sum of ladder diagrams is given by

$$
\chi_{\mathrm{pair}}^{\mathrm{ladder}}\left(\omega_{n}=0\right)=\sum_{m, n} \phi(m) A(I-A)^{-1}(m, n) .
$$

The identification of the pairing instability is to find the

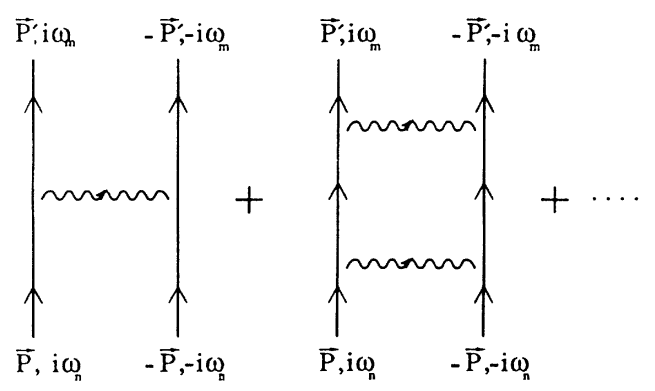

FIG. 3. The ladder approximation of the static pairing susceptibility. The straight line and wiggle line are electron Green's function and effective interaction $V_{\text {eff }}$ dressed with the impurities and phonons. temperature at which the matrix $A$ has the eigenvalue of unity.

The matrix $A(n, m)$ is real but nonsymmetric. For simplicity, we assume $Z_{n}$ is momentum independent. Integrating over the momentum, we have

$$
A(m, n)=-T \frac{\pi N(0)}{Z_{n}\left|\omega_{n}\right|} V_{\mathrm{eff}}\left(\omega_{m}, \omega_{n}\right),
$$

where $N(0)$ is the density of state at the Fermi level. For computational purpose, we transform the old basis $x_{n}$ of the matrix $A$ to the new basis $y_{n}$ such that the new matrix $B$ is real and symmetric. ${ }^{21}$ Let

$$
y_{n}=\frac{x_{n}}{\sqrt{Z_{n}\left|\omega_{n}\right|}},
$$

then $B$ is given by (see Owen and Scalapino ${ }^{21}$ )

$$
B(m, n)=-T \frac{\pi N(0)}{\sqrt{Z_{m} Z_{n}\left|\omega_{m} \omega_{n}\right|}} V_{\text {eff }}\left(\omega_{m}, \omega_{n}\right) .
$$

Now $B$ is symmetric because $V_{\text {eff }}\left(\omega_{m}, \omega_{n}\right)$ is symmetric. For a given set of parameters, the eigenvalue is invariant under similarity transformations, and hence $T_{c}$ is given by the highest temperature at which $B$ has largest eigenvalue 1 .

\section{B. $\left(\partial T_{c} / \partial c\right)_{c=0}$ formula}

To derive a formula for $\left(\partial T_{c} / \partial c\right)_{c=0}$, we follow Jarrell and start with

$$
B \mathbf{y}=e(T, c) \mathbf{y},
$$

with the conditions

$$
\begin{aligned}
& e\left(T_{c}, c\right)=1, \\
& \mathbf{y}^{T} \mathbf{y}=1 .
\end{aligned}
$$

Here $e(T, c)$ denotes the temperature- and concentration-dependent eigenvalue, and $\mathbf{y}$ is the corresponding eigenvector. Setting the total derivative of $e\left(T_{c}, c\right)$ to zero in the limit of $c \rightarrow 0$ gives

$$
\left(\partial T_{c} / \partial c\right)_{c=0}=\frac{-(\partial e / \partial c)_{c=0}}{\left(\partial e / \partial T_{c}\right)_{c=0}} .
$$

The normalization of the eigenvector implies

$$
\left(\frac{\partial e}{\partial c}\right)_{c=0}=\mathbf{y}^{T}\left(\frac{\partial B}{\partial c}\right)_{c=0} \mathbf{y}
$$

and

$$
\left(\frac{\partial e}{\partial T}\right)_{c=0, T=T_{c}}=\mathbf{y}^{T}\left(\frac{\partial B}{\partial T}\right)_{c=0, T=T_{c}} \mathbf{y} .
$$

In terms of the matrix $B$, the formula of $\left(\partial T_{c} / \partial\right)_{c=0}$ is given by

$$
\left(\partial T_{c} / \partial c\right)_{c=0}=\frac{-\mathbf{y}^{T}(\partial B / \partial c)_{c=0} \mathbf{y}}{\mathbf{y}^{T}\left(\partial B / \partial T_{c}\right)_{c=0} y} .
$$

Recall that $V_{\text {eff }}=V_{\mathrm{ph}}+V_{\mathrm{imp}}$; then $(\partial B / \partial c)_{c=0}$ is given by 


$$
\left[\frac{\partial B}{\partial c}\right]_{c=0}(m, n)=-\frac{T \pi N(0)}{\sqrt{\left|\omega_{m} \omega_{n}\right| Z_{m}^{0} Z_{n}^{0}}}\left[\Gamma\left(i \omega_{m}, i \omega_{n}\right)-\frac{1}{2}\left(\frac{\Sigma_{\mathrm{imp}}\left(i \omega_{m}\right)}{i \omega_{m} Z_{m}^{0}}+\frac{\Sigma_{\mathrm{imp}}\left(i \omega_{n}\right)}{i \omega_{n} Z_{n}^{0}}\right) V_{\mathrm{ph}}\left(i \omega_{m}, i \omega_{n}\right)\right],
$$

where $Z_{n}^{0}$ is the renormalization factor with no impurity contribution. If we define

$$
\eta_{n}=\frac{y_{n}}{T}\left(\frac{\pi\left|\omega_{n}\right|}{\left(\partial e / \partial T_{c}\right)_{c=0} Z_{n}^{0}}\right)^{1 / 2},
$$

and make use of Eqs. (37), (38), and (39) we have

$$
\begin{aligned}
\left(\partial T_{c} / \partial c\right)_{c=0}=\sum_{n, m} & \frac{\eta_{n} \eta_{m} T^{3} N(0) \Gamma(m, n)}{\left|\omega_{n} \omega_{m}\right|} \\
& -\sum_{n} \frac{\eta_{n}^{2} T^{2} \Sigma_{\text {imp }}\left(i \omega_{n}\right)}{\pi\left|\omega_{n}\right| i \omega_{n}} .
\end{aligned}
$$

The function $\eta_{n}$ approaches a constant of order unity for $\left|\omega_{n}\right| \ll<\omega_{0}$, and falls off as $\omega_{n}^{-2}$ for $\left|\omega_{n}\right| \gg>\omega_{0}$, thus providing a cutoff for $n$.

\section{TESTS OF THE CODES}

These tests on the quantum Monte Carlo code of the two-channel Kondo Hamiltonian and the code of calculating the change of superconducting critical temperature have been performed. Although many physical quantities of the two-channel Kondo impurity Hamiltonian have been calculated exactly by the Bethe ansatz, there are not many that can easily be reproduced by the quantum Monte Carlo method. The first test is to compare the on-site impurity spin susceptibility $\chi_{f f}$ with that calculated in the previous quantum Monte Carlo study of the single-channel Kondo impurity by Fye and Hirsch. ${ }^{36}$ To test the two-channel Kondo quantum Monte Carlo code, we set the impurity interaction in one of the channels to zero, and calculate the on-site impurity spin susceptibility.

Table I shows the results of impurity spin susceptibility $\chi_{f f}$ defined by

$$
\chi=-2 \int_{0}^{\beta} d \tau\left\langle T_{\tau} f_{\uparrow}^{\dagger}(\tau) f_{\downarrow}(\tau) f_{\downarrow}^{\dagger}(0) f_{\uparrow}(0)\right\rangle .
$$

The parameters chosen $(J=1.0$ and bandwidth $D=2)$ are identical to those of Fye and Hirsch. The second and the third columns are our data from quantum Monte Carlo

TABLE I. The inverse temperature $\beta$ dependence of the onsite spin susceptibility $\chi_{f f}$. The values of the second and the fourth columns are the result obtained by shutting off the impurity interaction of one channel in the two-channel Kondo impurities system, and the result of Hirsch and Fye in the singlechannel Kondo impurities system.

\begin{tabular}{lcccc}
\hline$\beta$ & $\chi_{f f}^{\mathrm{LJ}}$ & Error $(-/+)$ & $\chi_{f f}^{\mathrm{FH}}$ & Error $(-/+)$ \\
\hline 1 & 0.975 & 0.001 & 0.965 & 0.015 \\
2 & 1.806 & 0.004 & 1.800 & 0.03 \\
4 & 2.942 & 0.009 & 2.940 & 0.06 \\
8 & 4.067 & 0.018 & 4.080 & 0.32 \\
\hline \hline
\end{tabular}

calculations together with statistical errors, while the fourth and the fifth columns are those of Fye and Hirsch. We note that for a wide range of temperature ( $T=1.0$ to $T=0.125$ ), our results agree very well within the statistical error with those of Fye and Hirsch. In addition, the local static spin susceptibility measured with our Monte Carlo code shows the expected logarithmic divergence for $T \rightarrow 0 .{ }^{15,16}$ We have also compared the one-particle $t$ matrix for low Matsubara frequency and temperature with the result $t(\omega=0, T=0)=-\tau / 2 \pi N(0)$ anticipated from conformal field theory. ${ }^{3}$ We find perfect agreement to within statistical error. These two tests confirm that we are correctly sampling the nontrivial fixed point physics of the two-channel Kondo model with the quantum Monte Carlo code.

Having established the correctness of the two-channel Monte Carlo code, we proceed to test the $\left(\partial T_{c} / \partial c\right)_{c=0}$ code in the high-temperature limit $(T \gg J)$. The hightemperature test examines the effective interaction contribution to $\left(\partial T_{c} / \partial c\right)_{c}=0$. In the high-temperature limit, the impurity-induced effective electron-electron interactions calculated by the high-temperature perturbation theory are compared with those calculated by the $\left(\partial T_{c} / \partial c\right)_{c=0}$ code. We choose parameters $J=0.5$ and $N(0) J=0.25$ and compare the results of Monte Carlo with those of perturbation theory. An estimate of the Kondo temperature is about $0.0045 J$ for these parameters. For temperature $T=1$, we have $T / T_{k}=222$ and $J^{2} / T=0.25$. We thus believe that this is a reasonable high temperature. The next order of diagrams is the or-

TABLE II. The frequency $[\omega(n)=(2 n-1) \pi T]$ dependence of the impurity-induced effective electron-electron interaction for the flavor-singlet spin-triplet (top) and flavor-triplet spinsinglet (bottom) pairings in the two-channel Kondo impurities system. The second and fourth columns are the Monte Carlo results, while the third and fifth columns are the perturbation theory results. The parameters for exchange $J=0.5$, bandwidth $D=2$, and $T / T_{K}=222$.

\begin{tabular}{rrrcc}
\hline \multicolumn{1}{c}{$\omega$} & $\Gamma_{s t}^{\mathrm{mc}}(\omega, \omega)$ & $\Gamma_{s t}^{\text {pert }}(\omega, \omega)$ & $\Gamma_{s t}^{\mathrm{mc}}(\omega,-\omega)$ & $\Gamma_{s t}^{\text {pert }}(\omega,-\omega)$ \\
\hline-15.708 & -0.0150 & -0.0156 & 0.0010 & 0.000 \\
-9.424 & -0.0152 & -0.0156 & 0.0005 & 0.000 \\
-3.141 & -0.0160 & -0.0156 & 0.0020 & 0.000 \\
3.141 & -0.0160 & -0.0156 & 0.0020 & 0.000 \\
9.424 & -0.0152 & -0.0156 & 0.0005 & 0.000 \\
15.708 & -0.0150 & -0.0156 & 0.0010 & 0.000 \\
& & & & \\
$\omega$ & $\Gamma_{c t}^{\mathrm{mc}}(\omega, \omega)$ & $\Gamma_{c t}^{\text {pert }}(\omega, \omega)$ & $\Gamma_{c t}^{\mathrm{mc}}(\omega,-\omega)$ & $\Gamma_{c t}^{\text {pert }}(\omega,-\omega)$ \\
\hline 15.708 & 0.0170 & 0.0156 & 0.0299 & 0.0312 \\
-9.424 & 0.0152 & 0.0156 & 0.0303 & 0.0312 \\
-3.141 & 0.0150 & 0.0156 & 0.0286 & 0.0312 \\
3.141 & 0.0150 & 0.0156 & 0.0286 & 0.0312 \\
9.424 & 0.0152 & 0.0156 & 0.0303 & 0.0312 \\
15.708 & 0.0170 & 0.0156 & 0.0299 & 0.0312 \\
\hline \hline
\end{tabular}


der of $J^{4} / T^{3}=0.0625$, and yet the effective interaction is not too small to measure.

Table II shows the results of the Monte Carlo simulation and perturbation theory (to the order of $J^{2} / T$ ) for the effective interaction of flavor-triplet spin-singlet pairing and flavor-singlet spin-triplet pairing, respectively. We present the effective interaction values, in the frequency range, which contribute most to the change of critical temperature in the Eliashberg formalism. In both pairings, the signs and the magnitudes of the effective interaction agree well with the perturbation theory. The flavor-singlet spin-triplet interaction is attractive while the flavor-triplet spin-singlet interaction is repulsive. The inelastic effective interactions of flavor-singlet spin triplet in column 5 of Table II(top) are fluctuations which could be the contributions of the higher-order diagrams and it is an order lower than that of $J^{4} / T^{3}$. For both elastic interactions, the difference between the Monte Carlo and perturbation theory estimates is less than $8 \%$.

\section{RESULTS OF $\left(\partial T_{c} / \partial c\right)_{c}=0$}

In this section we present the results of $\left(\partial T_{c} / \partial c\right)_{c=0}$ for flavor-singlet spin-triplet and flavor-triplet spinsinglet pairings. The main results are that the induced electron-electron interaction for flavor-singlet spin-triplet pairing is attractive for all temperatures, while it is repulsive at high temperatures and attractive at low temperatures for flavor-triplet spin-singlet pairing. They all agree with the high-temperature perturbation theory and the low-temperature strong-coupling theory. ${ }^{37}$ However, $T_{c}$ is suppressed in both cases because of the strong pair breaking induced by the single-particle scattering.

The single-particle contribution suppresses the critical temperature for all temperatures because it is proportional to the absolute value of the $t$ matrix of conduction electrons. Physically, impurities destroy the quasiparticle states and decrease their lifetime. The effect is to suppress superconductivity.

Though we find enhancements in the critical temperature for the two-particle contributions with respect to the change in the concentration of impurities, the magnitude is very small, compared with the single-particle contribution for both flavor-singlet spin triplet and flavor-triplet spin singlet at all temperatures. The overall effect is critical temperature suppression at all temperatures.

We also find $\left(\partial T_{c} / \partial c\right)_{c=0}$ to be a universal function of $T_{K} / T_{c 0}$, i.e., $\left(\partial T_{c} / \partial c\right)_{c=0}$ can be expressed as the product of two universal functions $f\left(\lambda_{0}, \omega_{0}\right)$ and $g\left(T_{K} / T_{c 0}\right)$. Here $T_{c 0}$ is the critical temperature in the absence of impurities. The result is different from the critical temperature suppression in the Anderson model; $\left(\partial T_{c} / \partial c\right)_{c}=0$ can be expressed as a product of two universal functions $f\left(\lambda_{0}\right)$ and $g\left(T_{K} / T_{c 0}\right){ }^{22}$

\section{A. Pair-breaking contribution of $\left(\partial T_{c} / \partial c\right)_{c}=0$}

Figure 4 shows the contribution of the pair-breaking effect on $\left(\partial T_{c} / \partial c\right)_{c=0}$. The parameters $\omega_{0}=0.3$, $\lambda_{0}=2.07$, and $T_{c 0}=0.0357$ are chosen for the twochannel Kondo case, while the parameters $\omega_{0}=0.3$,

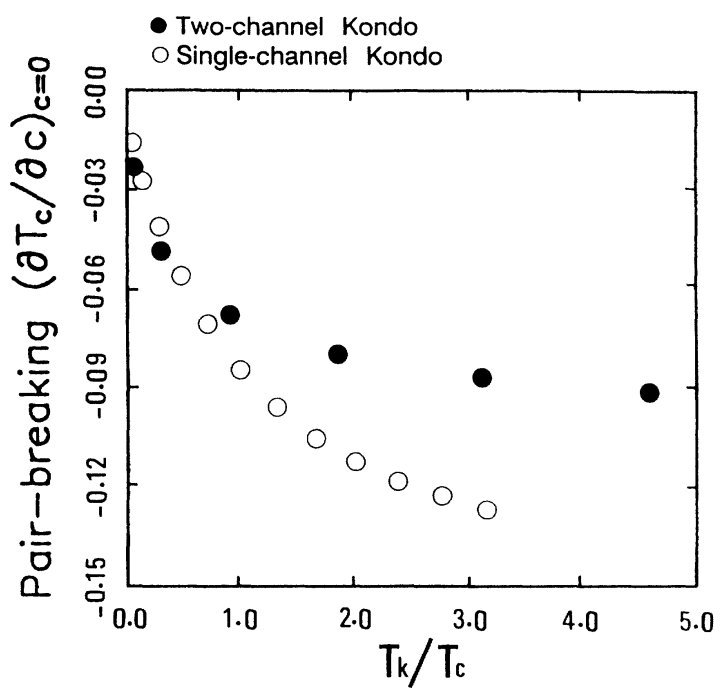

FIG. 4. The temperature dependence of pair-breaking contribution of $\left(\partial T_{c} / \partial c\right)_{c=0}$ in the two-channel Kondo and the single-channel Kondo impurities systems. For the two-channel Kondo impurities system, $\omega_{0}=0.3$ and $\lambda_{0}=0.0357$, while for the single-channel Kondo impurities system $\omega_{0}=0.3$ and $\lambda_{0}=3.9126$.

$\lambda_{0}=3.91$, and $T_{c 0}=0.0625$ are chosen for the singlechannel Kondo case. For the single-channel Kondo system, the pair-breaking effect increases with $T_{K} / T_{c 0}$. However, we expect that it will saturate to a constant value for $T_{K} / T_{c 0} \rightarrow \infty$. A weak-coupling calculation of $\left(\partial T_{c} / \partial c\right)_{c=0}$ on the resonant level model supports this expectation. ${ }^{38}$ However, a heuristic calculation at low $T_{c 0} \ll T_{K}$ was done by Matsuura, Ichinose, and Nagao$\mathrm{ka}^{23}$ and peaks were found in the single-particle and effective interaction contributions. Note that the work of Matsuura, Ichinose, and Nagaoka is exact in both limits $T_{c 0} \ll T_{K}$ and $T_{c 0} \gg T_{K}$, but is inaccurate for $T_{c 0} \sim T_{K}$. For the two-channel Kondo model we speculate that the pair-breaking effect persists even when the temperature goes to zero because the effective exchange grows to a finite value $J^{*}$, and the remaining effective spin breaks the Cooper pairs.

Indeed the renormalization-group picture qualitatively explains why the pair-breaking effect of the Kondo system is larger than the pair-breaking effect of the twochannel system in the intermediate region of $T_{K} / T_{c 0}$. The difference is that the fixed point of the two-channel Kondo model is at finite $J^{*}$ while the fixed point of the single-channel Kondo is at $J^{*}=\infty$. The electrons see a larger impurity potential for the single-channel Kondo model than the two-channel Kondo model for the same $T_{K} / T_{c}$, since the impurity spin of the single-channel Kondo model will be quenched gradually, while the impurity spin of the two-channel Kondo model will never be compensated as the temperature is lowered.

\section{B. Effective interaction contribution of $\left(\partial T_{c} / \partial c\right)_{c=0}$}

Figure 5 shows the two-particle contributions of $\left(\partial T_{c} / \partial c\right)_{c=0}$ for different $T_{K} / T_{c 0}$. The Einstein phonon 


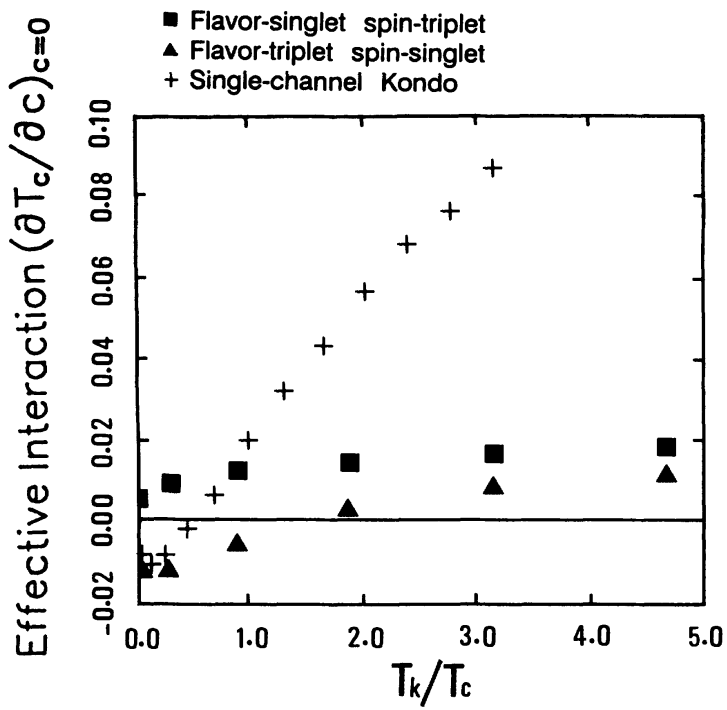

FIG. 5. The temperature dependence of effective interaction contribution of $\left(\partial T_{c} / \partial c\right)_{c=0}$ in the two-channel Kondo and the single-channel Kondo impurities systems. For the two-channel Kondo impurities system, $\omega_{0}=0.3$ and $\lambda_{0}=0.0357$, while for the single-channel Kondo impurities system $\omega_{0}=0.3$ and $\lambda_{0}=3.9126$.

frequency, electron-phonon coupling, and critical temperature are $\omega_{0}=0.3, \lambda=2.07$, and $T_{c 0}=0.0357$, respectively, for both sets of data. For flavor-singlet spin-triplet pairing an enhancement is found for all temperatures, while for flavor-triplet spin-singlet pairing suppression is found at high temperatures, and enhancement is found at lower temperatures. The crossover is at about the Kondo temperature and insensitive to the Einstein frequency. Note that the enhancement of $\left(\partial T_{c} / \partial c\right)_{c=0}$ for flavor-

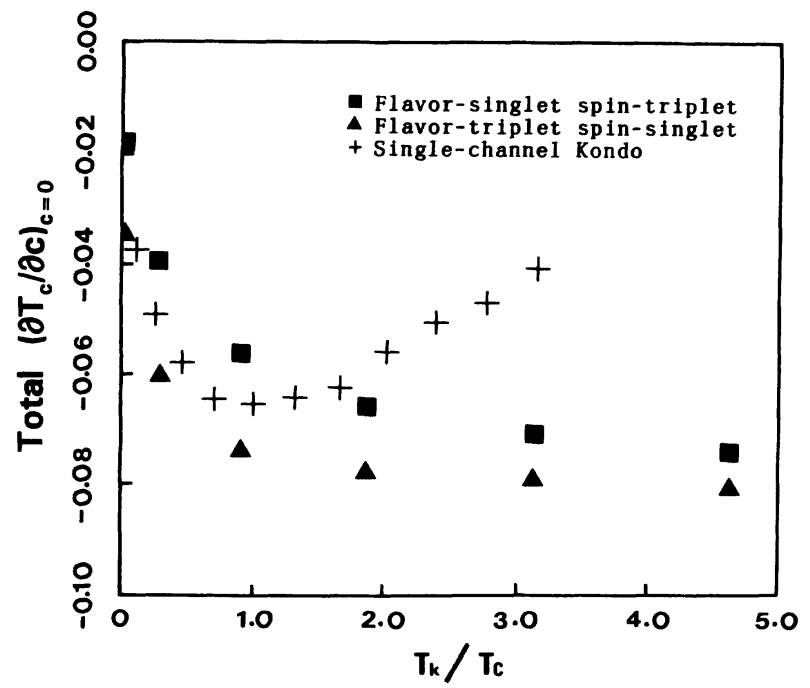

FIG. 6. The temperature dependence of $\left(\partial T_{c} / \partial c\right)_{c=0}$ in the two-channel Kondo and the single-channel Kondo impurities systems. For the two-channel Kondo impurities system, $\omega_{0}=0.3$ and $\lambda_{0}=0.0357$, while for the single-channel Kondo impurities system $\omega_{0}=0.3$ and $\lambda_{0}=3.9126$. singlet spin-triplet pairing is always greater than that of flavor-triplet spin-singlet. Such behavior is consistent with the renormalization-group picture. The enhancements saturate very quickly to finite values for both flavor-triplet spin-singlet and flavor-singlet spin-triplet pairings. We cautiously note that the fluctuation in the two-particle effective interaction is quite large, and we are unable to sample enough data for the quantity because of the limited amount of computer time, and thus the fluctuation is larger at lower temperature.

To get some insight, we compared the effective interaction contribution to $\left(\partial T_{c} / \partial c\right)_{c=0}$ of the two-channel Kondo system with that of single-channel Kondo system. The symbols + in Fig. 6 are the single-channel Kondo data with $\omega_{0}=0.3, \lambda_{0}=3.91$, and $T_{c 0}=0.0625$. We note that the qualitative features are preserved if we scale $\lambda_{0}$ to 2.07 , and thus it is meaningful to compare the data
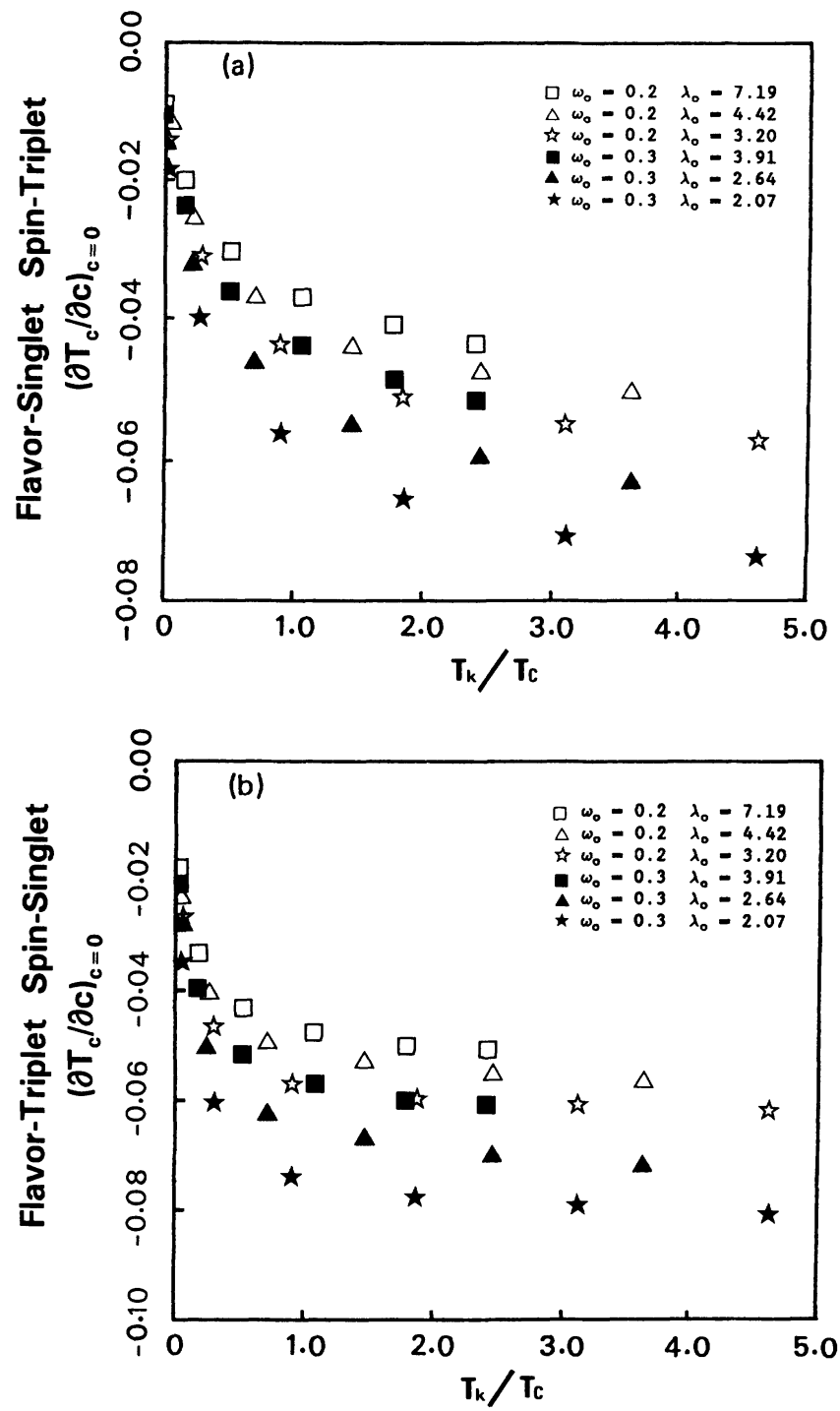

FIG. 7. The temperature dependence of $\left(\partial T_{c} / \partial c\right)_{c=0}$ for flavor-singlet spin-triplet (a) and flavor-triplet spin-singlet pairings (b) in the two-channel Kondo impurities system with Einstein phonon frequencies fixed. 
with those of the two-channel Kondo system (see Sec. VII D).

It is puzzling that the enhancement of the singlechannel Kondo system is much larger than that of the two-channel Kondo system below $T_{K} / T_{c 0}$. Physically, it is not clear that the effective interaction due to polarization for the single-channel Kondo system is much larger than that of two-channel Kondo system. However, the large enhancement of the single-channel Kondo system is consistent with the Anderson theorem of nonmagnetic impurity. For temperatures $T_{K} / T_{c 0} \gg 1$, the spin impurity of the single-channel Kondo system is essentially quenched, and it is thus nonmagnetic. As $T_{c} \rightarrow 0$, the two-particle effective interaction contribution has to compensate the single-particle pair-breaking contribution.
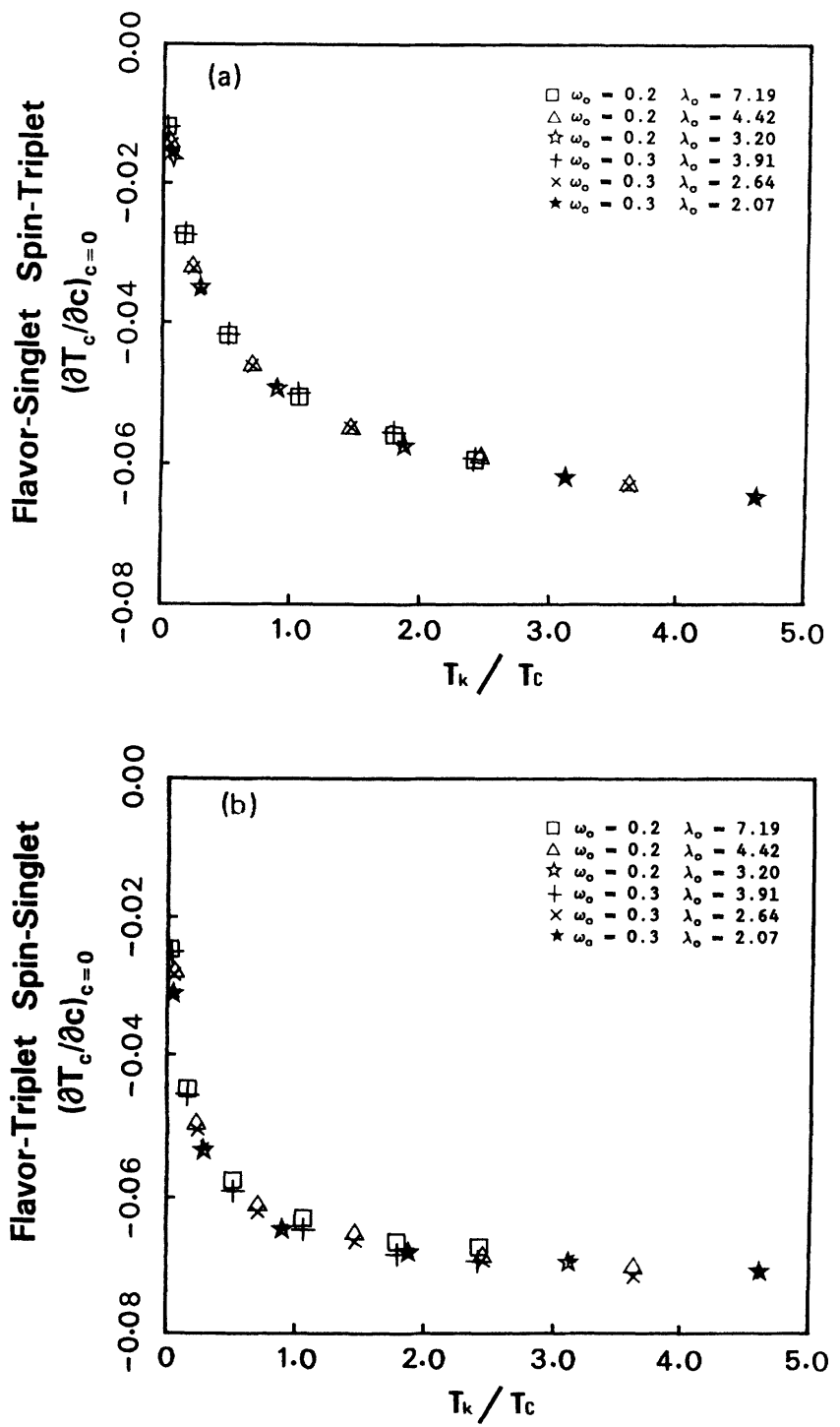

FIG. 8. The scaled temperature dependence of $\left(\partial T_{c} / \partial c\right)_{c=0}$ for flavor-singlet spin-triplet (a) and flavor-triplet spin-singlet pairings (b) in the two-channel Kondo impurities system with Einstein phonon frequencies fixed.
C. Total $\left(\partial T_{c} / \partial c\right)_{c=0}$

Figure 6 shows the total $\left(\partial T_{c} / \partial c\right)_{c=0}$ for the flavorsinglet spin-triplet and flavor-triplet spin-singlet pairings for the two-channel Kondo system. The Einstein phonon frequency, electron-phonon coupling, and critical temperature are $\omega_{0}=0.3, \lambda_{0}=2.07$, and $T_{c 0}=0.0357$, respectively, for both sets of data. The total $\left(\partial T_{c} / \partial c\right)_{c=0}$ for both flavor-singlet spin-triplet and flavor-triplet spinsinglet pairings are dominated by the single-particle pair-breaking contribution. We speculate that the saturation persists down to $T_{c 0} \ll T_{K}$ for both pairings since the effective spin remains at all temperature scales.

On the other hand, the $\left(\partial T_{c} / \partial c\right)_{c=0}$ data for the single-channel Kondo system have a peak right at
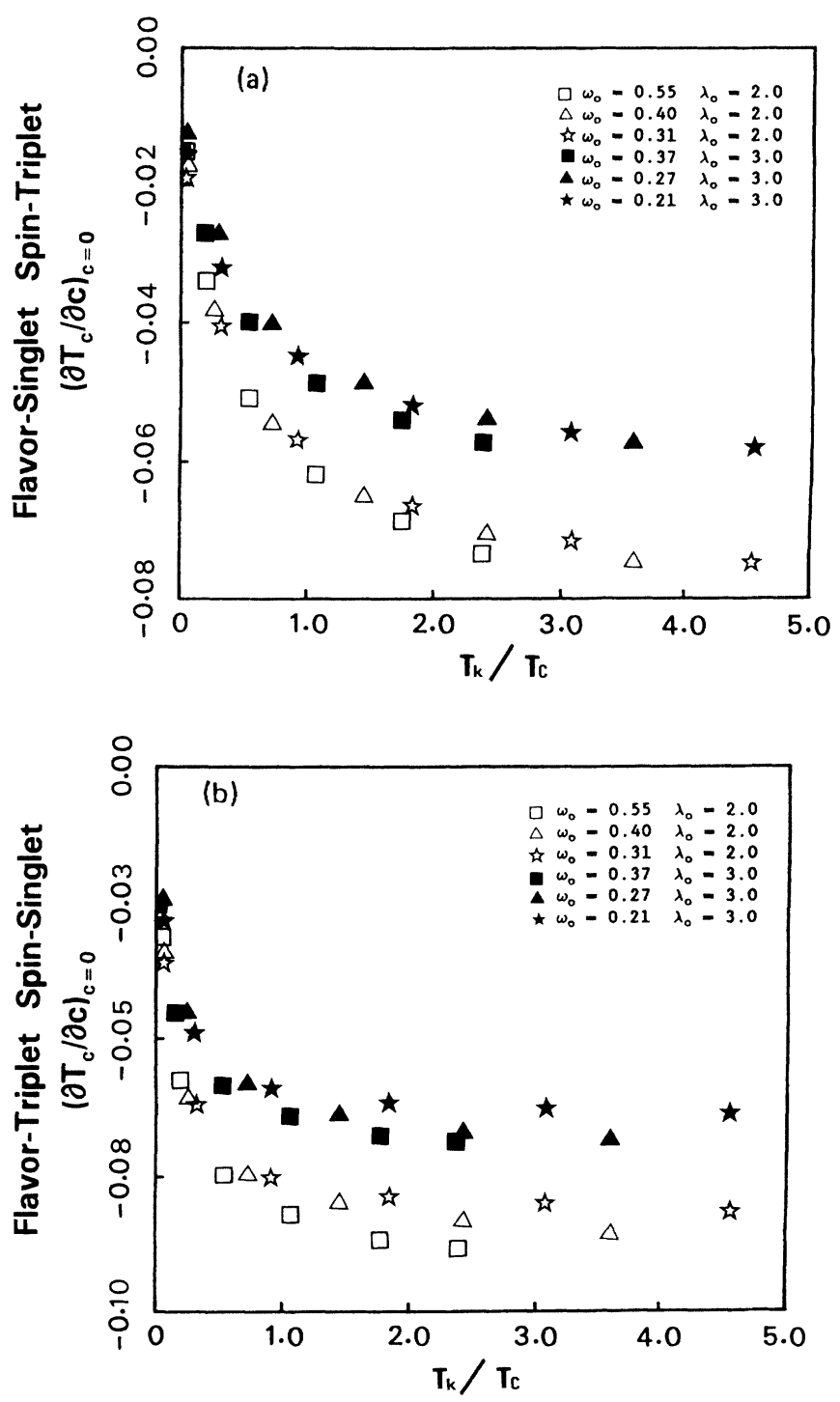

FIG. 9. The temperature dependence of $\left(\partial T_{c} / \partial c\right)_{c=0}$ for flavor-singlet spin-triplet (a) and flavor-triplet spin-singlet pairings (b) in the two-channel Kondo impurities system with electron-phonon coupling fixed. 
$T_{K}=T_{c 0}$ and start to go to zero for $T_{K}>T_{c 0}$, which is consistent with the Anderson theorem and the renormalization-group result that the effective exchange $J^{*}=\infty$. For these single-channel data, the Einstein phonon frequency, electron-phonon coupling, and critical temperature are $\omega_{0}=0.3, \lambda_{0}=3.91$, and $T_{c 0}=0.0625$, respectively.

\section{Universality $\left(\partial T_{c} / \partial c\right)_{c}=0$}

Both the flavor-singlet spin-triplet $\left(\partial T_{c} / \partial c\right)_{c}=0$ and flavor-triplet spin-singlet $\left(\partial T_{c} / \partial c\right)_{c=0}$ can be expressed empirically as a product of two universal functions $g\left(T_{K} / T_{c 0}\right)$ and $f\left(\lambda_{0}, \omega_{0}\right)$. The universality is demonstrated in two ways. First we fix the Einstein phonon frequency $\omega_{0}$, and adjust the electron-phonon coupling strength $\lambda_{0}$ such that the critical temperature in the ab-
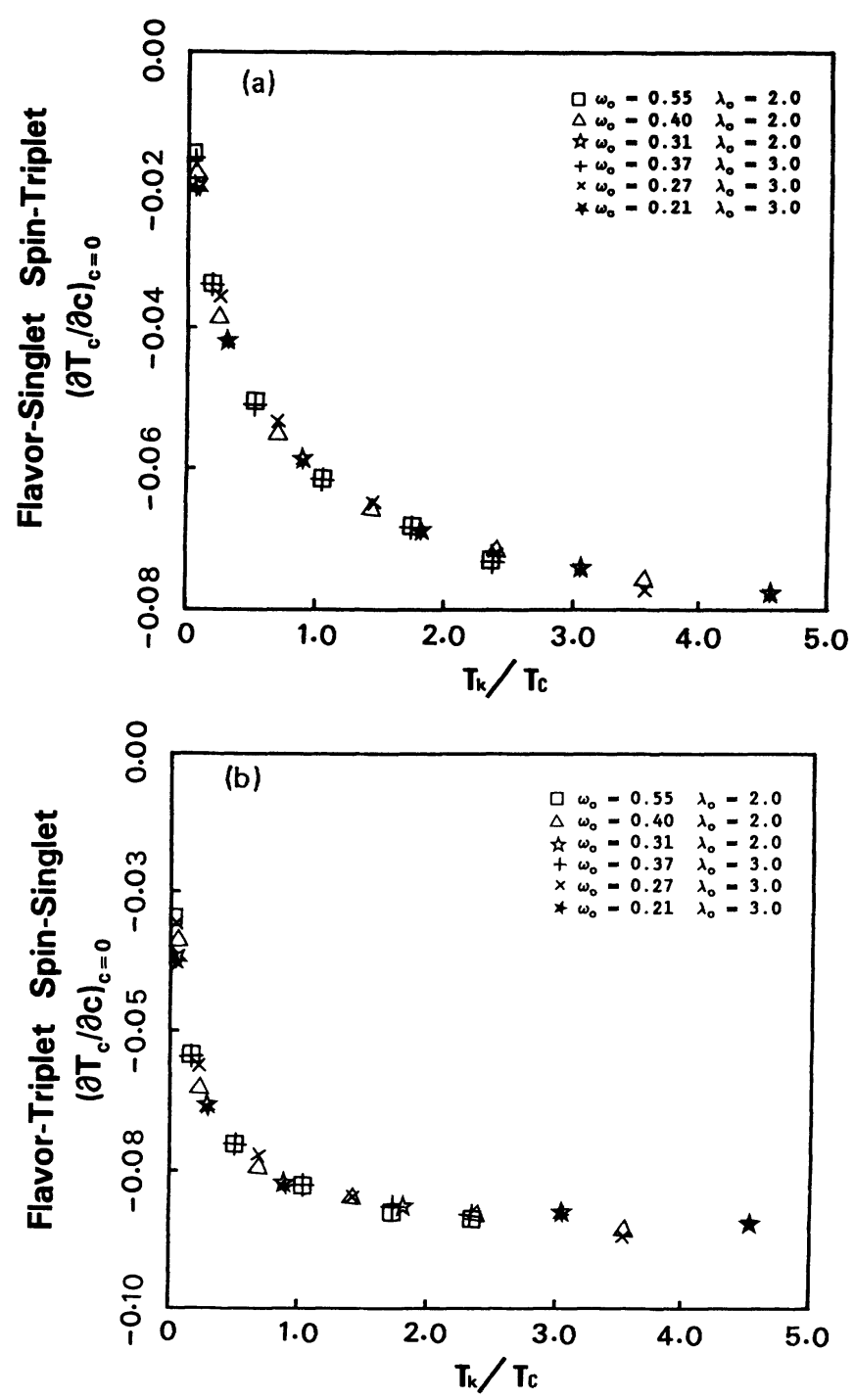

FIG. 10. The scaled temperature dependence of $\left(\partial T_{c} / \partial c\right)_{c=0}$ for flavor-singlet spin-triplet (a) and flavor-triplet spin-singlet pairings (b) in the two-channel Kondo impurities system with electron-phonon coupling fixed. sence of impurities $T_{c 0}$ is equal to the temperature $T$. Figures $7(a)$ and $7(b)$ show two sets of $\left(\partial T_{c} / \partial c\right)_{c=0}$ data for flavor-singlet spin-triplet and flavor-triplet spinsinglet pairings, respectively. Parameters for the first set are $\omega_{0}=0.2, \lambda_{0}=7.194, \omega_{0}=0.2, \lambda_{0}=4.416$, and $\omega_{0}=0.2$, $\lambda_{0}=3.204$, while parameters for the second set are $\omega_{0}=0.3, \lambda_{0}=3.914, \omega_{0}=0.3, \lambda_{0}=2.644$, and $\omega_{0}=0.3$, $\lambda_{0}=2.071$. The first and second sets of data can then be rescaled to overlap with the data set with parameters $\omega_{0}=0.3, \lambda_{0}=2.644$ by multiplication of constants, as shown in Figs. 8(a) and 8(b). The multiplication constants depend on $\lambda_{0}$ and $\omega_{0}$ only, but not on the pairing channels.

Second, we fix the electron-phonon coupling strength $\lambda_{0}$, and adjust the Einstein phonon frequency $\omega_{0}$ such that $T_{c 0}=T$. Figures $9(\mathrm{a})$ and $9(\mathrm{~b})$ show two sets of $\left(\partial T_{c} / \partial c\right)_{c=0}$ data for flavor-singlet spin triplet and flavor-triplet spin singlet, respectively. The first set $\left(\partial T_{c} / \partial c\right)_{c=0}$ parameters are $\lambda_{0} 2.0, \omega_{0}=0.545, \lambda_{0}=2.0$, $\omega_{0}=0.397$, and $\lambda_{0}=2.0, \omega_{0}=0.312$, while parameters for the second set are $\lambda_{0}=3.0, \omega_{0}=0.370, \lambda_{0}=3.0$, $\omega_{0}=0.269$, and $\lambda_{0}=3.0, \omega_{0}=0.211$. The first and second sets of data can then be rescaled to overlap with the data set of parameters $\lambda_{0}=2.0, \omega_{0}=0.397$ by multiplication of constants, as shown in Figs. 10(a) and 10(b). Again the multiplication constants depend on $\lambda_{0}$ and $\omega_{0}$ only, but not on the pairing channels. Hence, within the regions of $\omega_{0}$ and $\lambda_{0}$ explored in this paper, $\left(\partial T_{c} / \partial c\right)_{c=0}$ can be expressed as a product of two universal functions

$$
\left(\partial T_{c} / \partial c\right)_{c=0}=f\left(\lambda_{0}, \omega_{0}\right) g\left(T_{K} / T_{c 0}\right) .
$$

\section{CONCLUSION}

We have performed calculations on the change of the critical temperature of a superconducting host with respect to change in the concentration $c$ of the twochannel Kondo impurities in the limit of vanishing $c$. The host is characterized by two species of electron. The calculation is subjected to the Migdal theorem approximation and controllable statistical and systematic errors. The method is quantum Monte Carlo combined with Eliashberg formalism which takes the retardation effect of the electron-electron and electron-phonon interactions into account. The limitation of the quantum Monte Carlo method is that the calculation cannot be done at very low temperature.

We found for the temperature region $T_{K} / T_{c 0}<5$, the single-particle pair-breaking contribution of $\left(\partial T_{c} / \partial c\right)_{c}=0$ is a monotonic decreasing function of $T_{K} / T_{c 0}$. The effective interaction contribution of $\left(\partial T_{c} / \partial c\right)_{c=0}$ enhances $T_{c}$ for flavor-singlet spin-triplet pairings, while it suppresses $T_{c}$ at high temperatures and enhances $T_{c}$ at low temperatures for flavor-triplet spin-singlet pairing. For both flavor-singlet spin-triplet and flavor-triplet spin-singlet pairings, $T_{c}$ suppression is found, and $\left(\partial T_{c} / \partial c\right)_{c=0}$ saturates quickly when $T_{K} / T_{c 0}>1$. There is no maximum in $\left(\partial T_{c} / \partial c\right)_{c=0}$ for $T_{K} / T_{c 0}<5$, unlike the single-channel Kondo impurity model. Within the 
parameter ranges we explored, $\left(\partial T_{c} / \partial c\right)_{c=0}$ for flavorsinglet pairing and flavor-triplet spin-singlet pairing can be universally expressed as a product of two functions $g\left(T_{K} / T\right)$ and $f\left(\lambda_{0}, \omega_{0}\right)$, where $\lambda_{0}$ and $\omega_{0}$ are the electron-phonon coupling constant and Einstein frequency, respectively.

Among the extensions of this work worth exploring are (i) calculation of $T_{c}$ at finite concentrations and (ii) calculation of $T_{c}$ in the odd-in-time-reversal channel referred to in the Introduction, assuming the host metal possesses odd-in-time-reversal pairing.

\section{ACKNOWLEDGMENTS}

This work was supported by research funding from the U.S. Department of Energy, Office of Basic Energy Sciences, Division of Materials Research. We thank the Ohio Supercomputer Center for a grant of time allowing these computations to be carried out. K.-H.L. thanks Dr. P. M. Hui for a critical reading of the manuscript and for the hospitality during his stay at CUHK, while D.L.C. and M.J. acknowledge stimulating discussions with H.-B. Pang.
${ }^{1}$ P. Nozieres and A. Blandin, J. Phys. (Paris) 41, 193 (1980).

${ }^{2}$ L. L. Hirst, Z. Phys. 11, 255 (1970).

3(a) A. W. W. Ludwig and I. Affleck, Phys. Rev. Lett. 33, 3160 (1991); (b) A. W. W. Ludwig, Physica B 199\&200, 406 (1994).

${ }^{4}$ V. Emery and S. Kivelson, Phys. Rev. B 46, 10812 (1992).

${ }^{5}$ J. Gan, N. Andrei, and P. Coleman, Phys. Rev. Lett. 70, 868 (1993).

${ }^{6}$ D. L. Cox and A. E. Ruckenstein, Phys. Rev. Lett. 71, 1613 (1993).

${ }^{7}$ C. L. Seaman, M. B. Maple, B. W. Lee, S. Ghamaty, M. S. Torikachvili, J.-S. Kang, L. Z. Liu, J. W. Allen, and D. L. Cox, Phys. Rev. Lett. 67, 2882 (1991).

${ }^{8}$ H. Amitsuka, T. Hidano, T. Henna, H. Mitamura, and T. Sakakibara, Physica B 186-188, 337 (1993).

${ }^{9}$ B. Andraka, Phys. Rev. B 49, 3589 (1994).

${ }^{10}$ D. C. Ralph and R. A. Buhrman, Phys. Rev. Lett. 69, 2118 (1992).

${ }^{11}$ D. C. Ralph, J. von Delfft, R. A. Buhrman, and A. W. W. Ludwig, Phys. Rev. Lett. 72, 1064 (1994).

${ }^{12}$ D. L. Cox, Phys. Rev. Lett. 59, 1240 (1987).

${ }^{13}$ D. L. Cox, Physica B 186-188, 312 (1993).

${ }^{14}$ A. Zawadowski, Phys. Rev. Lett. 45, 211 (1980).

${ }^{15}$ P. D. Sacramento and P. Schlottmann, Phys. Lett. A 142, 245 (1989).

${ }^{16}$ P. D. Sacramento and P. Schlottmann, Phys. Rev. B 40, 431 (1989).

${ }^{17}$ D. L. Cox, Physica C 153, 1624 (1988).

${ }^{18}$ A. M. Tsvelik, J. Phys. Condens. Matter 2, 2833 (1990).

${ }^{19}$ H.-B. Schüttler, M. Jarrell, and D. J. Scalapino, J. Low Temp. Phys. 69, 159 (1987).
${ }^{20}$ H.-B. Schüttler, M. Jarrell, and D. J. Scalapino, Phys. Rev. Lett. 58, 1147 (1987).

${ }^{21}$ C. Owen and D. J. Scalapino, Physica 55, 691 (1971).

${ }^{22}$ M. Jarrell, Phys. Rev. Lett. 61, 2612 (1988).

${ }^{23}$ M. Jarrell, Ph.D. thesis, University of California, Santa Barbara, 1987 (unpublished).

${ }^{24}$ T. Matsuura, S. Ichinose, and Y. Nagaoka, Prog. Theor. Phys. 57, 713 (1976).

${ }^{25}$ D. J. Scalapino, in Superconductivity, edited by R. D. Park (Marcel Dekker, New York, 1969), Vol. 2, p. 449.

${ }^{26}$ D. J. Scalapino, J. R. Schrieffer, and J. W. Wilkins, Phys. Rev. B 148, 263 (1966).

${ }^{27}$ A. B. Migdal, Sov. Phys. JETP 7, 966 (1958).

${ }^{28}$ G. D. Mahan, Many-Particle Physics (Plenum, New York, 1986).

${ }^{29}$ G. D. Mahan, Many-Particle Physics (Ref. 28), p. 243.

30J. E. Hirsch and R. M. Fye, Phys. Rev. Lett. 56, 2521 (1986).

${ }^{31}$ J. R. Schrieffer and P. A. Wolff, Phys. Rev. B 149, 491 (1966).

${ }^{32}$ C. Kittel, Quantum Theory of Solids (Wiley, New York, 1987), p. 152.

${ }^{33}$ J. W. Negele and H. Orland, in Quantum Many-Particle Systems, edited by D. Pines (Addison-Wesley, New York, 1988), p. 105.

${ }^{34}$ L. D. Landau, Sov. Phys. JETP 5, 101 (1957); 6, 920 (1957); 35, 70 (1959).

$35 \mathrm{~J}$. W. Negele and H. Orland, in Quantum Many-Particle Systems (Ref. 33), p. 100.

${ }^{36}$ R. M. Fye and J. E. Hirsch, Phys. Rev. B 40, 4780 (1989).

${ }^{37}$ K.-H. Luk, Ph.D. thesis, The Ohio State University, 1992.

${ }^{38}$ D. L. Cox (private communication). 